\title{
Resultados da descompressão e anteriorização do nervo ulnar no cotovelo
}

\section{Results of ulnar nerve descompression and anterior transposition at the elbow}

Trabalho realizado na Rede Sarah de Hospitais, Brasília, DF, Brasil.

Artigo submetido pelo SGP (Sistema de Gestão de Publicações) da RBCP.

Artigo recebido: 3/1/2011 Artigo aceito: 15/2/2011

\begin{abstract}
RESUMO
Introdução: A compressão do nervo ulnar no cotovelo é a segunda causa mais frequente de neuropatia compressiva no membro superior. Na maioria dos casos, a compressão ocorre no canal cubital, vulnerável à compressão extrínseca, intrínseca ou idiopática. O tratamento cirúrgico é controverso. Método: Os autores descrevem os resultados da descompressão e transposição anterior do nervo ulnar realizadas em 58 pacientes. Resultados: Identificou-se como causa principal a fratura de cotovelo e a hanseníase. Na Rede Sarah, entretanto, têm sido frequente $(15 \%)$ também as indicações por compressão causada por ossificação heterotópica em casos de lesados medulares e/ou cerebrais. Realizamos $57 \%$ de procedimentos associados à retinaculotomia dos flexores, descompressão no canal de Guyon e transferência tendínea para músculos intrínsecos. Obteve-se $80 \%$ de bons e excelentes resultados, $20 \%$ de complicações, dor neuropática, distrofia simpática reflexa e manutenção dos sintomas, observadas em pacientes diabéticos e portadores de hanseníase.
\end{abstract}

Descritores: Síndromes de Compressão Nervosa. Síndrome do Túnel Ulnar. Nervo Ulnar.

\begin{abstract}
Introduction: Ulnar nerve compression is the second most frequent entrapment neuropathy of the upper extremity. The most cases to occur at the elbow, with an extrinsic, intrinsic or idiopathic compression. The surgical technique varies. Methods: This study assesses the results of a series of subcutaneous transpositions of the ulnar nerve. Results: The most frequent cause is elbow fracture, and Hansen disease. In the Sarah Network is frequent heterotopic ossification entrapment because spinal cord injury and stroke. We performed $57 \%$ associated surgical procedures for Guyon, carpal decompression and tendinous transference. This study shows $80 \%$ cases were good and excellent results and $20 \%$ of complications, neuropathy pain, complex regional pain and persistent symptoms in diabetes and Hansen disease.
\end{abstract}

Keywords: Nerve Compression Syndromes. Cubital Tunnel Syndrome. Ulnar Nerve.

1. Membro titular; Médica, cirurgiã plástica do Hospital Sarah Brasília, Brasília, DF, Brasil.

2. Membro titular; Médico, cirurgião plástico do Hospital Sarah Brasília, Brasília, DF, Brasil.

3. Doutor em Ortopedia; Cirurgião-chefe da Rede Sarah de Hospitais, Brasília, DF, Brasil. 


\section{INTRODUÇÃO}

A compressão do nervo ulnar é a segunda causa mais frequente de neuropatia compressiva. Ocorre no cotovelo, na arcada de Struthers, no septo intermuscular, no epicôndilo medial, na cabeça medial do tríceps, no túnel cubital com a banda de Osborne e na aponeurose do pronador e flexores, arcada do flexor ulnar do carpo ${ }^{1-3}$, resultando em isquemia e neurite crônica. A sua etiologia inclui anormalidades ósseas, fraturas, tumores, gânglios, constrição fascial, subluxação do nervo no epicôndilo medial durante os movimentos de flexão do cotovelo e ossificação heterotópica ${ }^{4,5}$. O diagnóstico é clínico e complementado pelo exame eletroneuromiográfico $^{1-3,6}$.

O tratamento conservador pode ser efetivo, e quando não há melhora clínica realiza-se a descompressão do nervo ulnar no canal cubital. Em 1930, Learmonth ${ }^{7}$ popularizou a técnica para descompressão e transposição do nervo ulnar no cotovelo. Desde então, vários procedimentos têm sido descritos, muito embora, com controvérsias quanto a melhor opção.

O objetivo desse artigo é descrever os resultados da descompressão e transposição subcutânea anterior do nervo ulnar, realizados pela Equipe de Cirurgia Plástica do Hospital Sarah Brasília, durante seis anos.

\section{MÉTODO}

Os pacientes submetidos a descompressão e transposição anterior do nervo ulnar no cotovelo, operados no Hospital Sarah Brasília, entre 2001 a 2007, foram avaliados quanto à idade, ao sexo e à causa. Os sintomas e sinais sensitivos, motores, subjetivos e objetivos foram obtidos no pré e pós-operatório, conforme a classificação de McGownan ${ }^{2,4}$ modificada (Quadro 1). O programa Epi-info versão 3.4.3 foi utilizado para análise dos dados.

Quadro 1 - Classificação de McGownan ${ }^{4}$ modificada para compressão de nervo ulnar no cotovelo avaliados no préoperatório.

\begin{tabular}{c|c|c|c}
\hline & Grau I & Grau II & Grau III \\
\hline & $\begin{array}{c}\text { Sintomas } \\
\text { subjetivos } \\
\text { somente }\end{array}$ & $\begin{array}{c}\text { IIa sintomas objetivos } \\
\text { sem atrofia da } \\
\text { musculatura intrínseca } \\
\text { da mão IIb com } \\
\text { atrofia de musculatura } \\
\text { intrínseca da mão grau } \\
\text { M3 -M4 }\end{array}$ & $\begin{array}{c}\text { Disfunção } \\
\text { sensitiva e } \\
\text { motora com } \\
\text { paralisia da } \\
\text { musculatura } \\
\text { intrínseca da } \\
\text { mão }\end{array}$ \\
\hline $\mathrm{N}$ & 2 & 38 & 18 \\
\hline
\end{tabular}

A cirurgia foi indicada para os pacientes que apresentavam: persistência dos sintomas após tratamento conservador (medidas de proteção, uso de órtese, amitriptilina e antinflamatório, por 4 a 12 meses); achados objetivos da redução da sensibilidade avaliada pelo Teste de Semmeis-Weiss e motora, conforme a Medical Research Council; exame eletroneuromiográfico confirmando o comprometimento do nervo ulnar no segmento do canal cubital e exame de imagem evidenciando compressão extrínseca, como nos casos de hanseníase e ossificação heterotópica (Figuras 1 e 2).

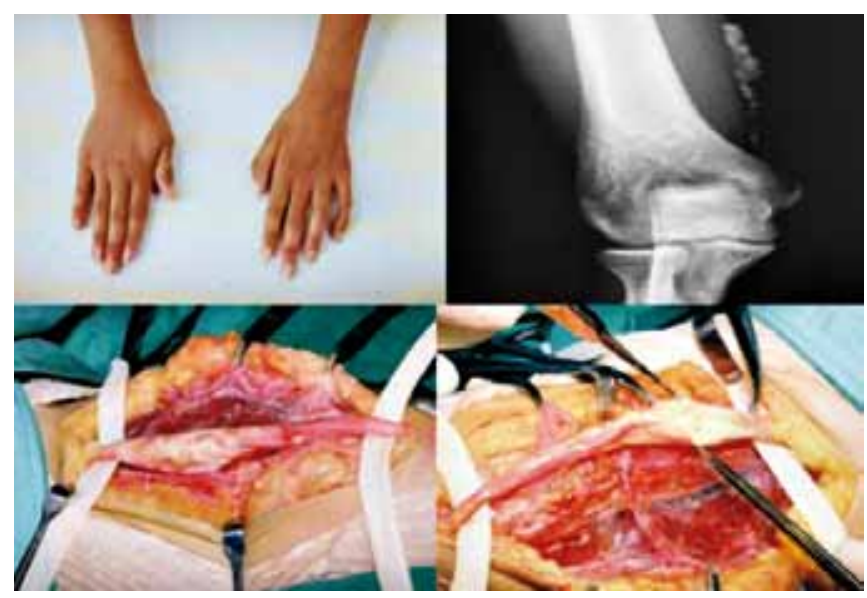

Figura 1 - Vista de paciente no pré-operatório com sinais de paralisia do nervo ulnar; exame radiológico e transoperatório apresentando abscesso caseoso por neurite hansênica do nervo ulnar. Transoperatório de descompressão e anteriorização do nervo ulnar no canal cubital.

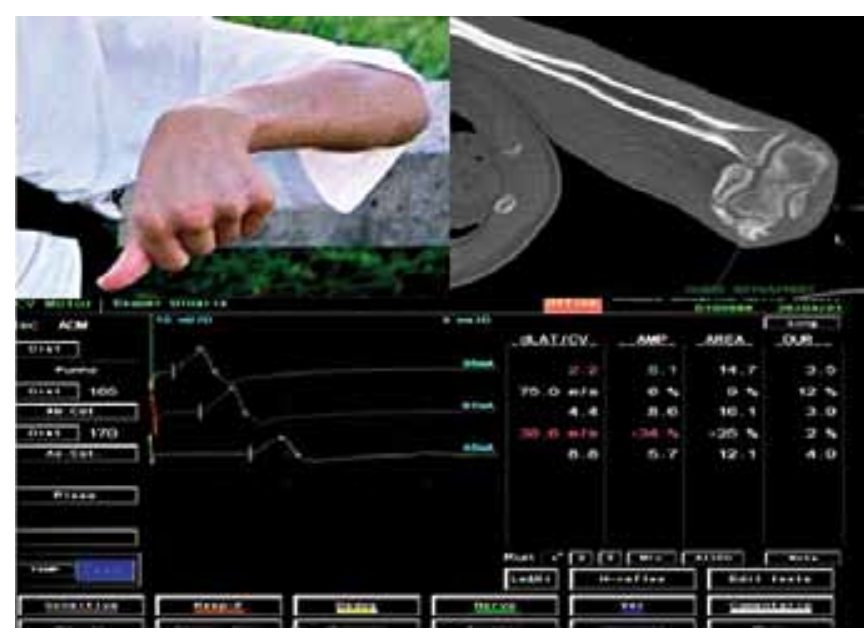

Figura 2 - Paciente portador de sequela de trauma cranioencefálico apresentava deformidade em flexão do cotovelo devido a ossificação heterotópica associado à compressão do nervo ulnar no canal cubital visto ao exame radiológico do cotovelo e no estudo da condução motora do nervo ulnar. 


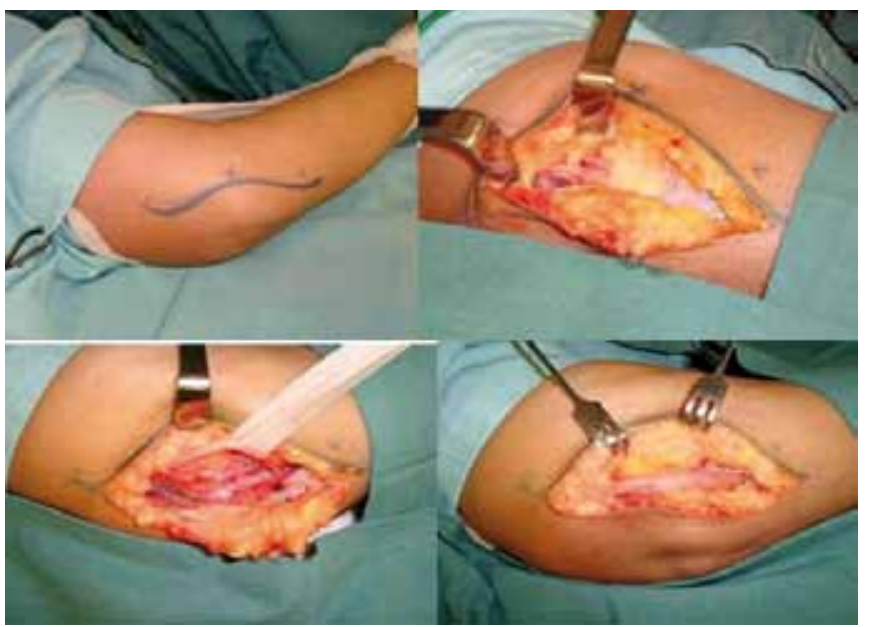

Figura 3 - Sequência operatória: marcação, descompressão e transposição anterior do nervo ulnar no cotovelo.

\section{Técnica Cirúrgica}

Os pacientes foram submetidos à anestesia bloqueio de plexo, axilar ou geral e ao esvaziamento do membro superior com faixa de Esmarch. Incisão de dez a $12 \mathrm{~cm}$ foi realizada na porção medial do cotovelo (Figura 3). Dissecção dos tecidos e do nervo proximal e distal, preservando-se o ramo cutâneo medial do antebraço e os ramos do nervo ulnar. Após a abertura do septo intermuscular e arcada de Struthers, realizou-se a transposição anterior no subcutâneo, preservando-se a vascularização. Ao final, flexionou-se o cotovelo para avaliar eventuais constrições ao movimento ou luxação do nervo. Procedeu-se à sutura subcutânea com monocryl 3-0 e imobilização com tala posterior, mantendo o cotovelo flexionado durante duas a três semanas.

\section{Pós-operatório}

Os pacientes foram acompanhados no programa fisioterápico e ambulatorial, utilizou-se o protocolo de $\mathrm{McGownan}^{4}$ no pré e no pós-operatório, em média após um ano. Foram afastados do trabalho por, no mínimo, oito semanas.

\section{RESULTADOS}

Foram estudados 58 pacientes entre 2001 e 2007, seis operados bilateralmente. Todos apresentavam neuropatia compressiva do nervo ulnar no cotovelo, evidenciaramse alterações subjetivas, objetivas e foram submetidos à descompressão e transposição anterior no subcutâneo.

Um paciente abandonou o tratamento no terceiro mês de pós-operatório. O tempo de seguimento foi em média de um ano (seis meses a seis anos). Observou-se o predomínio no sexo feminino, entre 40-60 anos de idade (Figura 4).

A causa da compressão foi fratura de cotovelo em onze pacientes, hanseníase em nove, ossificação heterotópica em portadores de lesões cerebrais e/ou medulares em nove, compressão extrínseca por gota ou ganglion e não identificada em oito pacientes (Figura 5). Outras síndromes compressivas foram encontradas, tais como a síndrome do túnel do carpo em 18 pacientes e do canal de Guyon, em nove.

O critério para elegibilidade do procedimento cirúrgico baseou-se na persistência dos sintomas de parestesia e dor, apesar do tratamento conservador e/ou do tempo de evolução da compressão, nos achados objetivos (alteração sensitiva e motora) e nos resultados do exame eletromiográfico, sendo este último considerado menos importante na indicação cirúrgica, muito embora utilizado para confirmação diagnóstica da compressão neural no cotovelo, com achados mielínicos e/ou axonais. Em relação aos resultados do exame eletroneuromiográfico realizado entre 8 e 12 meses de pós-operatório, identificou-se melhora em 28 pacientes, com a normalização no exame em 12, inalterado em 12, sequelar em 5 e piora em 3 , não realizado nos demais. Os casos que apresentaram piora tinham diagnóstico de hanseníase.

A biopsia do epineuro evidenciou processo inflamatório inespecífico em 38 (64\%) pacientes, 10 (17\%) apresentavam fibrose, três ( $5 \%$ ) hanseníase, um caso de hemorragia recente do nervo, um de processo granulomatoso, um de tofo gotoso (Figura 6) e um de ganglion.

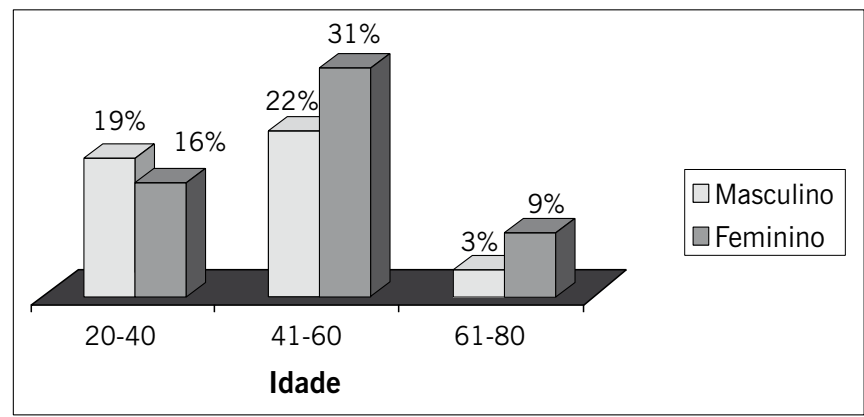

Figura 4 - Distribuição quanto ao sexo e à idade.

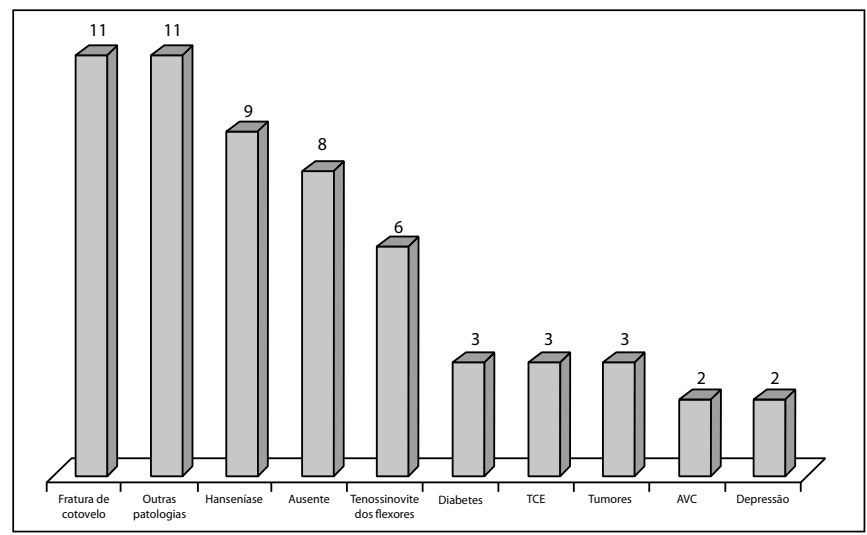

Figura 5 - Condições associadas. 


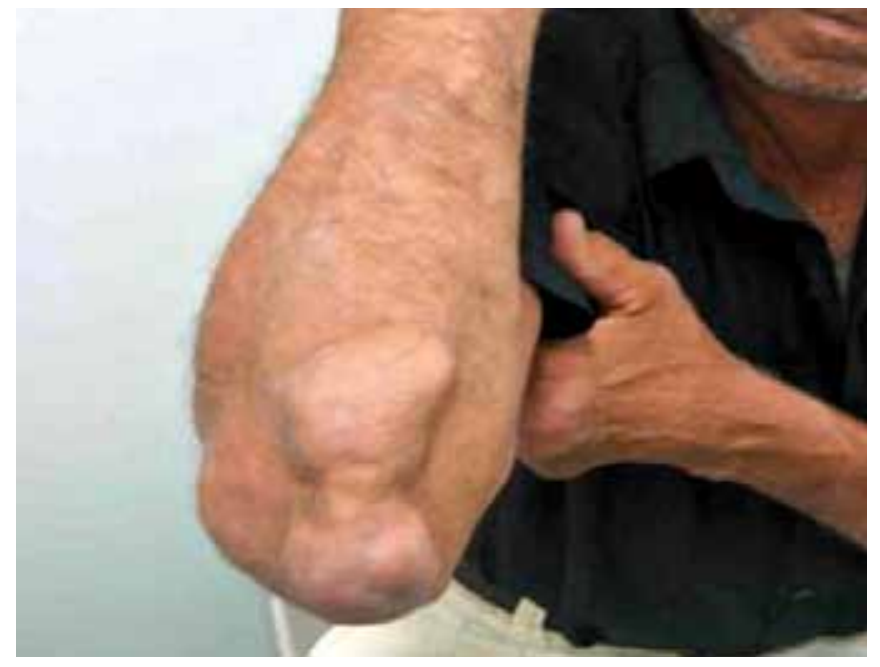

Figura 6 - Paciente apresentando compressão extrínseca do nervo ulnar por tofo gotoso no cotovelo.

A maioria dos pacientes (56 casos) foi classificada no pré-operatório nos Graus II e III de McGownan ${ }^{4}$ (Tabela 1). Esses pacientes apresentavam alterações objetivas da sensibilidade e motora no pré-operatório, constrição do nervo no canal cubital observada no transoperatório. Dos nove pacientes diagnosticados como hanseníase, oito apresentavam Grau III.

\begin{tabular}{|c|c|c|}
\hline \multicolumn{3}{|c|}{$\begin{array}{c}\text { Tabela } 1 \text { - Distribuição dos procedimentos cirúrgicos } \\
\text { associados realizados. }\end{array}$} \\
\hline Cirurgia & Frequência & $\%$ \\
\hline Descompressão e transposição anterior & 16 & $28 \%$ \\
\hline Ressecção de ossificação heterotópica & 9 & $16 \%$ \\
\hline RTC + abertura no canal de Guyon + biopsia & 6 & $10 \%$ \\
\hline Biopsia & 5 & $9 \%$ \\
\hline Retinaculotomia dos flexores (RTC) & 5 & $9 \%$ \\
\hline Transferência tendínea & 3 & $5 \%$ \\
\hline $\begin{array}{l}\text { Retinaculotomia dos flexores }+ \text { abertura de } \\
\text { polias A1 }\end{array}$ & 3 & $5 \%$ \\
\hline Retinaculotomia dos flexores + biopsia & 2 & $3 \%$ \\
\hline Abertura de polia A1 & 2 & $3 \%$ \\
\hline $\begin{array}{l}\text { Guyon + Retinaculotomia dos flexores }+ \\
\text { biopsia }\end{array}$ & 1 & $2 \%$ \\
\hline $\begin{array}{l}\text { Retinaculotomia dos flexores }+ \\
\text { transferência tendínea }\end{array}$ & 1 & $2 \%$ \\
\hline Ressecção de cisto no cotovelo & 1 & $2 \%$ \\
\hline Retirada de tofo no cotovelo & 1 & $2 \%$ \\
\hline $\begin{array}{l}\text { Abertura do primeiro compartimento } \\
\text { extensor }\end{array}$ & 1 & $2 \%$ \\
\hline Guyon + biopsia & 1 & $2 \%$ \\
\hline Guyon + biopsia bilateral & 1 & $2 \%$ \\
\hline Total & 58 & $100 \%$ \\
\hline
\end{tabular}

Em $28 \%$ dos casos, realizou-se somente a descompressão e a transposição anterior e, em 35 (57\%), foram realizados procedimentos associados: biopsia de epineuro, ressecção de ossificação heterotópica, descompressão no canal de Guyon e do nervo mediano no canal carpiano e outros procedimentos (abertura de compartimento extensor, abertura de polia) (Tabela 2).

Em 53\% dos pacientes, observou-se a normalização dos sintomas e sinais apresentados no pré-operatório e, em 16 (27,6\%), melhora em relação ao pré-operatório (Grau I). O procedimento foi efetivo em $80 \%$ dos casos (Tabela 3) e $20 \%$ apresentaram como complicações dor neuropática, dor complexa regional e persistência dos sintomas (Tabela 4). Em quatro daqueles pacientes classificados como Grau III, realizou-se a transferência tendínea, utilizando-se o tendão flexor superficial do IV ou V, conforme a Cirurgia do Laço de Zancolli, obtendo-se melhora da função da mão (Tabela 5).

Tabela 2 - Resultados obtidos após a descompressão e transposição anterior do nervo ulnar no cotovelo.

\begin{tabular}{l|c|c}
\hline Resultados & $\begin{array}{c}\text { Pós- } \\
\text { operatório }\end{array}$ & $\%$ \\
\hline Grau I - sintomas subjetivos somente & 16 & $27,6 \%$ \\
\hline $\begin{array}{l}\text { Grau II - fraqueza muscular ou } \\
\text { alterações sensitivas objetivas (a)sem } \\
\text { atrofia muscular ou com (b)atrofia inicial }\end{array}$ & 8 & $13,8 \%$ \\
\hline $\begin{array}{l}\text { Grau III - atrofia muscular, alteração } \\
\text { motora e sensitiva }\end{array}$ & 2 & $3,4 \%$ \\
\hline Normal & 31 & $53,4 \%$ \\
\hline Não avaliado & 1 & $1,7 \%$ \\
\hline Total & 58 & $100,0 \%$ \\
\hline
\end{tabular}

\begin{tabular}{|c|c|c|c|c|c|c|}
\hline $\begin{array}{l}\text { Pré/Pós- } \\
\text { operatório }\end{array}$ & Grau I & $\underset{\text { II }}{\text { Grau }}$ & $\begin{array}{c}\text { Grau } \\
\text { III }\end{array}$ & Normal & $\begin{array}{c}\text { Não } \\
\text { avaliado }\end{array}$ & Total \\
\hline Grau I & 0 & 0 & 0 & $2(100 \%)$ & 0 & 2 \\
\hline Grau II & $8(21 \%)$ & $1(3 \%)$ & 0 & $28(73 \%)$ & $1(3 \%)$ & 38 \\
\hline Grau III & $8(44 \%)$ & $7(40 \%)$ & $2(11 \%)$ & $1(6 \%)$ & 0 & 18 \\
\hline Total & 16 & 8 & 2 & 31 & 1 & 58 \\
\hline
\end{tabular}

Tabela 4 - Distribuição das complicações.

\begin{tabular}{l|c|c}
\hline Complicações & Frequência & $\mathbf{\%}$ \\
\hline Dor neuropática & 6 & $10,3 \%$ \\
\hline Distrofia Simpático-Reflexa tipo I & 5 & $6,9 \%$ \\
\hline Persistência dos sintomas e sinais & 1 & $1,7 \%$ \\
\hline Total & 12 & $20 \%$ \\
\hline
\end{tabular}


Tabela 5 - Resultados obtidos após a descompressão e transposição anterior do nervo ulnar no cotovelo quanto à avaliação sensitiva, conforme o Teste de Semmeis-Weiss.

\begin{tabular}{l|c|c}
\hline \multirow{2}{*}{ Avaliação sensitiva } & \multicolumn{2}{c}{ Frequência } \\
\cline { 2 - 3 } & Pré & Pós \\
\hline S4 verde - 1,65-2,83 - normal & 14 & 47 \\
\hline S3 + azul - 3,22-3,61 - toque leve diminuído & 22 & 4 \\
\hline S2 púrpura - 3,84-4,31 - sensação protetora diminuída & 2 & 5 \\
\hline S1 vermelho - 4,56-6,65 - perda de sensação protetora & 10 & 3 \\
\hline S0 $>$ 6,65 - anestesia & 3 & 2 \\
\hline disestesia & 6 & 6 \\
\hline Não avaliado & 1 & 1 \\
\hline Total & 58 & 58 \\
\hline
\end{tabular}

\section{DISCUSSÃO}

O tratamento cirúrgico da neuropatia compressiva do nervo ulnar no cotovelo é ainda controverso, possível de recidivas ou mesmo de falha ${ }^{1,3}$. Os procedimentos cirúrgicos descritos são descompressão, transposição e epicondilectomia ${ }^{1-3}$. Em uma revisão sistemática, Dellon ${ }^{5}$ revisou 50 artigos, totalizando 2000 pacientes operados, observou que a preferência pessoal da equipe permanece como principal critério para seleção do procedimento cirúrgico. Nas compressões moderadas e graves, a descompressão e a transposição muscular oferecem os melhores resultados, com menos recorrência. Segundo Nathan et al. ${ }^{8}$, a descompressão simples é um procedimento relativamente descomplicado, que preserva a anatomia normal, mas com maior suscetibilidade a traumatismos e alongamento do nervo e possibilidade de subluxação em $2,4 \%$ dos casos. Nos casos de epicondilectomia, Popa \& Dubert ${ }^{1}$ descreveram como vantagens a preservação do suprimento sanguíneo e dos tecidos em torno do nervo, mas pode ocorrer a instabilidade medial devido à desinserção do ligamento colateral ântero-medial, a perda da proteção do epicôndilo medial, a dor no pós-operatório devido a osteotomia, a subluxação do nervo sobre o epicôndilo e a contratura em flexão do cotovelo.

O procedimento descrito nesse estudo foi preconizado em $1930^{7}$, com modificações. Partindo da nossa experiência e de outros autores, empregou-se a descompressão e a transposição do nervo ulnar no tecido subcutâneo. Essa técnica tem sido defendida por alguns autores ${ }^{13}$, devido à melhora clínica que apresenta e criticada por outros pela desvascularização que proporciona no nervo ${ }^{9,10} \mathrm{e}$ à possibilidade de criar um novo sítio de compressão. Entretanto, é possível preservar a vascularização extrínseca do nervo ${ }^{11,12} \mathrm{e}$, tendo-se o cuidado e habilidade técnica, também, prevenir nova compressão. Em relação à fibrose proporcionada no nervo, consideramos que todas as técnicas evoluem com algum grau de fibrose devido ao descolamento do nervo. A qualidade do resultado depende do tempo do início dos sintomas e da etiologia ${ }^{8,9}$. As complicações observadas foram a dor neuropática e a distrofia simpático-reflexa, sobretudo em pacientes portadores de diabetes mellitus e mal de Hansen. Observaram-se seis casos de disestesia sem distrofia simpático reflexa, possivelmente devido à lesão por tração do ramo cutâneo medial do antebraço, nenhum paciente se queixou da posição mais superficial do nervo e quatro apresentaram a manutenção dos sintomas compressivos do nervo semelhante ao que é apresentado na literatura ${ }^{8-10}$. Vale ressaltar nessa casuística a frequência de procedimentos cirúrgicos associados, dentre esses a ressecção de ossificação heterotópica, cujo resultado foi de ganho funcional de amplitude de flexo-extensão do cotovelo e melhora clínica.

Os autores concordam que realizar a descompressão simples tornaria o nervo mais suscetível, passível de compressão, de traumatismos e manteria o alongamento do nervo durante os movimentos de flexão do cotovelo. Todavia, a utilização da descompressão e anteriorização do nervo ulnar no cotovelo reduz o alongamento no seu sulco ${ }^{14}$, consequentemente diminuindo a tensão e a neurite. Além disso, foi possível $80 \%$ de bons e excelentes resultados em pacientes classificados como grau II e III.

\section{CONCLUSÃO}

A descompressão e a anteriorização do nervo ulnar permitiram o alívio dos sintomas e sinais subjetivos e objetivos de neuropatia compressiva do nervo ulnar no canal cubital em $80 \%$ dos pacientes. As complicações foram observadas em $20 \%$ dos pacientes e relacionadas na maioria dos casos à hanseníase.

\section{REFERÊNCIAS}

1. Popa M, Dubert T. Treatment of cubital tunnel syndrome by frontal partial medial epicondylectomy. A retrospective series of 55 cases. J Hand Surg. 2004;29(6):563-7.

2. Lascar T, Laulan J. Cubital tunnel syndrome: a retrospective review of 53 anterior subcutaneous transpositions. J Hand Surg. 2000;25(5):453-6.

3. Hoffmann R, Siemionow M. The endoscopic management of cubital tunnel syndrome. J Hand Surg. 2006;31(1):23-9.

4. McGowan AJ. The results of transposition of the ulnar nerve for traumatic ulnar neurttis. J Bone Joint Surg. 1950;32-B(3): 293-301.

5. Dellon AL. Review of treatment results for ulnar entrapment at the elbow. J Hand Surg Am. 1989;14(4):688-700.

6. Paz AC, Carod Artal FJ, Kalil RK. A function of proprioceptors in bone organization: a possible explanation for neurogenic heterotopic ossification in patients with neurological damage. Med Hypotheses. 2007;68(1):67-73.

7. Learmonth JR. A technique for transplanting the ulnar nerve. Surg Gynecol Obstet. 1942;75:792-3. 
8. Nathan PA, Myers LD, Keniston RC, Meadows KD. Simple decompression of the ulnar nerve: an alternative to anterior transposition. J Hand Surg Br. 1992;17(3):251-4.

9. Lundborg G. Surgical treatment for ulnar nerve entrapment at the elbow. J Hand Surg Br. 1992;17(3):245-7.

10. Manske PR, Johnston R, Pruitt DL, Strecker WB. Ulnar nerve decompression at the cubttal tunnel. Clin Orthop Relat Res. 1992;(274):231-7.

11. Lebreton E, Bourgeon Y, Lascombes P, Merle M, Foucher G. Systematisation de la vaseulartsation de la portton brachmle du neff ulnmre. Annal Chir la Mare. 1983;2:211-8.
12. Rogers MR, Bergfield TG, Aulicino PL. The failed ulnar nerve transposition. Etiology and treatment. Clin Orthop Relat Res. 1991;(269):193-200.

13. Macadam SA, Gandhi R, Bezuhly M, Lefaivre KA. Simple descompression versus anterior subcutaneous and submuscular transposition of the ulnar nerve for cubital tunnel syndrome: a meta-analysis. J Hand Surd Am. 2008;33(8):1314.e1-12.

14. Grewal R, Varitimidis SE, Vardakas DG, Fu FH, Sotereanos DG. Ulnar nerve elongation and excursion in the cubital tunnel after descompression and anterior transposition. J Hand Surg Br. 2000;25(5):457-60.

\section{Correspondência para:}

E-mail: katiatb@terra.com.br 